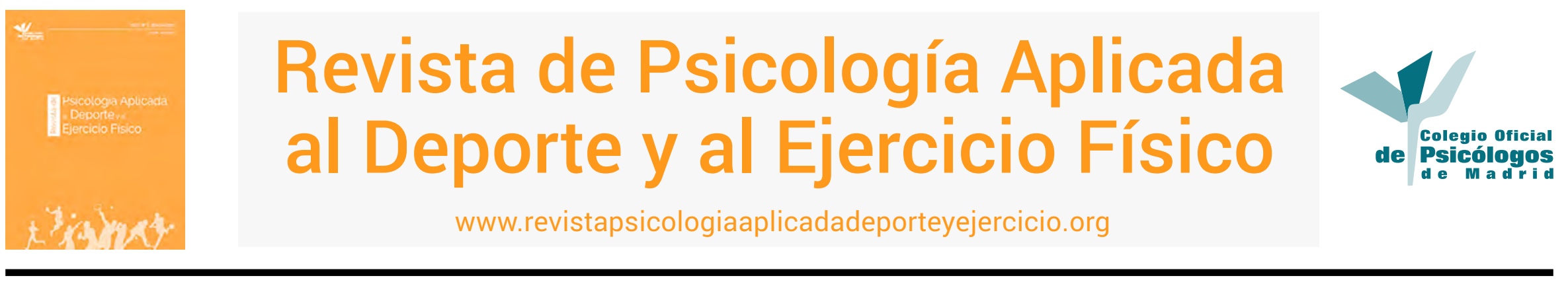

\title{
"No todo es Balón". Implicando positivamente a los padres en la práctica del fútbol en etapa formativa
}

\author{
Paula Ortiz Marholz \\ Escuelas Deportivas UCfútbol Quilicura, Chicureo, Independencia, Usach
}

RESUMEN: Los padres cumplen un rol fundamental en el desarrollo personal de sus hijos en el ámbito del deporte y en la vida en general, aunque no siempre actúan de la mejor manera ni toman las mejores decisiones. Este trabajo pretende hacer un análisis teóricopráctico sobre las actitudes de los padres en el deporte, plantear los comportamientos que se asocian positivamente a la motivación, el disfrute y el compromiso deportivo de los niños. Por otro lado, se presenta el trabajo psicológico realizado con padres de alumnos entre 7-12 años, así como la labor desempeñada con los propios niños y entrenadores en una escuela de fútbol en Chile. Como resultados más significativos se observa el posicionamiento positivo del rol del psicólogo del deporte, la mejora de la autonomía y el disfrute de los alumnos, la valoración del esfuerzo sobre el resultado y evaluar el error como parte del aprendizaje (por parte de los niños y sus padres), generar espacios de reflexión acerca del rol de los padres y el liderazgo positivo de los entrenadores hacia el desarrollo deportivo y crecimiento personal de los alumnos.

PALABRAS CLAVES: Fútbol formativo, motivación de logro, valores, padres.

\section{"The ball is not everything". Positive involvement of parents in the practice of football in the formative stage}

ABSTRACT: Parents play an essential role in personal development of their children in the field of sports and in life in general, but they do not always act in the best way or make the best decisions. This paper aims to make a theoretical-practical analysis of the attitudes of parents in sport, to consider the behaviors that are positively associated with the motivation, enjoyment, and sports commitment of children. The psychological work done with parents of students aged 7-12 years, and with the children and coaches in a soccer school in Chile, is also reported. The most significant results include the positive positioning of the role of the sports psychologist, improvements in children autonomy and enjoyment, assessment of the effort on the result and evaluation of error as part of learning (by children and their parents), generation of spaces for reflection about the role of parents, and the positive leadership of coaches with regard to sports development and personal growth of students.

KEYWORDS: Formative football, achievement motivation, values, parents.

\section{Overtraining e esporte sob uma perspectiva psicológica: estado em questão}

RESUMO: A atividade física moderada tem inúmeros benefícios para a saúde física e mental, mas à medida que a quantidade de tempo de dedicação e carga de trabalho durante o treinamento aumentam, pode causar alguns sintomas ou problemas de saúde, incluindo diferentes estados de fadiga que podem levar à Síndrome do Overtraining (SSE). Os objetivos deste artigo são estabelecer uma distinção conceitual entre a SSE e outros conceitos relacionados, analisar como é avaliada para detecção e analisar estudos representativos sobre o tema publicado entre 2010 e 2017. Conclui-se que os sintomas relacionados com a SSE são muito variados (mudanças orgânicas, psicológicas, comportamentais, etc.) e podem apresentar diferenças dependendo da modalidade esportiva (maior em esportes individuais), nível de competição (maior em alto desempenho) e até mesmo de uma pessoa para outra. Geralmente, é detectada quando há sintomas óbvios e está avançada, como o declínio do desempenho atlético, embora seu início seja anterior. Portanto, é necessário um estudo individualizado da SSE, no qual é recomendada a detecção precoce e o acompanhamento periódico,

Paula Ortiz Marholz es Psicóloga en la Escuelas Deportivas UCfútbol Quilicura, Chicureo, Independencia, Usach.

La correspondencia acerca de este artículo debe enviarse a Paula Ortiz Marholz, c/ Los Prados 21. Condominio Los Candiles. Piedra Roja, Chicureo. Colina, Santiago de Chile. e-mail:

(cc) EY-No-No Este es un artículo Open Access bajo la licencia 
sendo o POMS um dos instrumentos mais utilizados e efetivos devido à variação do humor relacionado com a síndrome. Além disso, a um nível prático, é importante rever e ajustar os tempos de dedicação, cargas de treinamento e recuperação entre as sessões do atleta, bem como o descanso, o sono e a alimentação. O psicólogo do esporte pode assessorar e orientar os técnicos esportivos sobre a SSE, avaliar e intervir psicologicamente para sua prevenção e tratamento.

PALAVRAS-CHAVE: Futebol educativo, motivação de realização, valores, pais.

Artículo recibido: 05/05/2017 | Artículo aceptado: 09/07/2017

Se ha podido observar e investigar que gran parte de los niños y adolescentes realizan alguna práctica deportiva de forma regular, ya sea dentro del marco escolar o fuera de él (García-Ferrando, 2006). La literatura científica avala que la actividad física y el deporte en sí no generan compromiso ni intención de practicarlo sostenidamente entre los niños y adolescentes, así como tampoco promueve automáticamente el bienestar físico y psicológico (Duda y Balaguer, 2007; Quested y Duda, 2011). En relación a esto, Roberts y Treasure (2012) han sugerido que es la calidad de los entornos sociales, creada por otros significativos (como los padres y entrenadores), que generan implicaciones y consiguientes respuestas psicológicas de los deportistas. Se supone que estas respuestas psicológicas son determinantes críticos de la participación deportiva a largo plazo y conduce a la salud física y mental (Jones, Dunn, Holt, Sullivan y Bloom, 2009; Quested et al., 2014).

Los padres cumplen roles fundamentales en el desarroIlo de sus hijos (Määttä, Ray y Roos, 2014; Merino, Arraiz y Sabirón, 2017; Smith, Cumming y Smoll, 2008). Como cualquier tarea, esos roles pueden ser cumplidos de manera satisfactoria o, sin juzgar intención, no traducirse en efectos positivos para los hijos. Se ha dicho "sin juzgar intención" porque se puede suponer que los padres son las primeras personas en querer lo mejor ara sus hijos, en querer que éstos desarrollen al máximo sus capacidades y fortalezas, y sin embargo, se cae en muchos errores, por ejemplo decir verbalmente que el resultado es lo importante y al perder un partido, no hablarle o tener un lenguaje no verbal de molestia, o compararlo constantemente con los compañeros, estar pendiente del ranking, etc. Estas conductas muchas veces generan frustraciones y efectos no deseados tanto en sus hijos como en ellos mismos.

Los padres, puede reflejar expectativas, emociones, pasiones e incluso proyectar en los hijos sus frustraciones (Torregrosa, Chamorro y Ramis, 2016). Por ejemplo, contribuyen a desarrollar conceptos como el significado del "éxito" y muchas veces basando su autoconcepto y valía personal en la retroalimentación recibida por los adultos significativos. García Mas et al. (2011), planteó que el contexto influye en un atleta y esa influencia es dada por la forma cómo ese contexto es interpretado subjetivamente en cuanto a criterios presentes de éxito y fracaso. Este ambiente reflejará las acciones de entrenadores y padres, así como el uso que hagan de recompensas, castigos y retroalimentación. En esta línea, el trabajo de Baumrind (1971) fue uno de los primeros trabajos en examinar la relación padres e hijos y determinó que a los niños que se les concedió más oportunidades por sus padres a la libre determinación, el autocontrol, participación en el diseño de la tarea y que se vieron reforzados, desarrollaron una mayor sensación de autonomía, que se tradujo en un mayor rendimiento académico.

En el ámbito deportivo, se encuentra en la teoría de orientación motivacional de logro, una propuesta que tiene como objetivo el análisis de los distintos factores disposicionales y ambientales que influyen en la motivación del deportista (Ames, 1992; Newton, Duda, y Yin, 2000; Nicholls, 1989). Según este constructo teórico, los factores disposicionales hacia la tarea (esfuerzo, superación y aprendizaje autoreferenciado) o el ego (búsqueda de resultados y superar a los demás, tomando como referente lo externo) reflejan el criterio por el cual los deportistas juzgan su nivel de competencia y por el que, subjetivamente, definen el éxito o el fracaso en el deporte. Por otro lado, los factores ambientales se refieren al conjunto de señales generadas por familiares, amigos, entrenador, etc., y percibidas por el deportista en el entorno, a través de las cuales se define las claves del éxito y del fracaso y generan el clima motivacional. En función de cómo percibe el deportista el contexto habría un clima motivacional implicado hacia el ego o la tarea (Ames, 1992).

La influencia de los padres en el desarrollo de la motivación de logro de los niños se basa en el argumento de que las orientaciones de meta se desarrollan a través de experiencias de socialización en la infancia y se supone que los padres juegan un papel muy importante en el desarrollo de estas preferencias e interpretación del éxito (Nicholls, 1989; Roberts, 2001).

Una investigación realizada por Wuerth, Lee, y Alfermann (2004) concluyeron que el apoyo que reciben los hijos de los padres influye notablemente en su satisfacción y percepción 
de competencia deportiva. Es así, que la actitud y el interés que muestren los padres hacia la actividad deportiva pueden influir en la decisión del hijo, su grado de implicación y el enfoque que se le da a la misma (Fredericks y Eccles, 2006), viendo que los deportistas pueden variar sus orientaciones disposicionales de meta en función de sus experiencias de socialización en el contexto deportivo (Castillo, Duda, Álvarez, Mercé y Balaguer, 2011; Gómez-López, Granero-Gallegos, Baena-Extremera y Abraldes, 2014).

Como se ha expuesto, existen investigaciones que han mostrado que comportamientos de apoyo y de comprensión de los padres se asocian a percepciones positivas con la actividad, así como también con el aumento de sensaciones de disfrute (Merino et al., 2017). Así se ha visto que los padres a través de la generación de climas orientados a la tarea, pueden influir en la generación de metas centradas en el aprendizaje y no en el resultado (Veskovic, Valdevit y Dordevic-Kikic, 2013). En este sentido, O’Rourke, Smith y Smoll (2014) encontraron que el clima motivacional iniciado por los padres fue un significativo predictor al final de la temporada sobre el autoestima, ansiedad rasgo y la regulación autónoma por sobre el clima iniciado por el entrenador. Por el contrario, presiones de los padres por el resultados se asocian a una disminución del disfrute y sensaciones de amotivación (Sánchez, Leo, Sánchez-Oliva, Amado y García, 2013).

Desde el punto de vista disposicional, ambas orientaciones de meta no son dicotómicas desde una perspectiva estadística, sino ortogonales entre sí (Nicholls, 1989), es decir cómo explica Cervelló, Escartí y Balagué (1999), cuando medimos la orientación motivacional, podemos llegar a encontrarnos con deportistas que estén orientados a la tarea y al ego simultáneamente, variando en la intensidad del patrón motivacional. Los deportistas pueden variar sus orientaciones disposicionales de meta en función de sus experiencias de socialización en el contexto deportivo (Gómez-López et al., 2014). De acuerdo a esto y a últimos estudios que combinan perfiles de orientación tarea y ego (Ortiz, 2016), se ha indicado que en climas altos en tarea y bajo en ego respaldan un clima motivacional de aprendizaje y disfrute. Por el contrario, climas de alto-ego y baja-tarea sienten que su madre y su padre valoran un clima donde el éxito se asoció con poco esfuerzo y sintieron que sus padres les hicieron preocupan por cometer errores. A su vez, en climas de alta-tarea y alto-ego, se encontró asociación con la percepción de padres que relacionaban el éxito con bajos niveles de esfuerzo ejercido y a madres que mostraban preocupación por cometer errores. Sin embargo, también se encontró en el grupo de alto-tarea y alto-ego que ambos padres valoran el aprendizaje y el disfrute en el desarrollo de habilidades físicas. Finalmen- te, los individuos que mostraron bajo-tarea y bajo-ego percibieron que sus madres les hacían sentir miedo de cometer errores en el aprendizaje de habilidades.

Se conoce, por tanto, que las percepciones del clima motivacional creado por padres y entrenadores generan un gran impacto en el desarrollo de habilidades físicas, emocionales y sociales de los jóvenes deportistas (Taliaferro, Rienzo, y Donovan, 2010; Ortiz, Chirosa, Martín, Reigal y García-Mas, 2016). De este modo, el entorno deportivo se convierte en un medio de influencias socializadoras que representan un papel importante en la formación de las características personales del niño (Torregrosa et al., 2007).

Finalmente y de acuerdo a la literatura (Cerezo, Casanova, De la Torre y De la Villa, 2011; Ortiz et al., 2016) los padres son modelos de comportamiento, y por tanto, lo que se haga y lo que se deje de hacer tienen efectos, ya sea positivos o negativos. También hay que tener presente que es todo un entorno el que influye en esta vivencia: entrenadores, pares, infraestructura, directivos, planificación de los programas, familiares, espectadores, etc. Sin embargo y a la luz de todo lo expuesto, la función de los padres, sobre todo en etapas de iniciación y formativas, parece ser fundamental para el desarrollo positivo de los niños con el deporte y para ello, al igual que todos los actores involucrados en la actividad, deben poder "educarse" de qué trata todo este mundo del deporte, para así mostrar actitudes que ayuden a que sus hijos obtengan beneficios a través de la práctica. Como pueden ser asegurarse que los profesionales a cargo del programa donde se encuentren sus hijos, están capacitados y el lugar sea de calidad; mantener comunicación abierta y positiva con los profesores o entrenadores; apoyar las actividades del club o escuela; otorgar las posibilidades para un buen desarrollo, ya sea traslados o cumplir con los compromisos económicos adquirido; mostrar estar interesados y preguntar a sus hijos cómo estuvo la práctica: cómo lo han pasado y si les gusta, eso mostrará valor a la actividad misma y el disfrute.

\section{Experiencia Profesional}

En la práctica profesional se pueden observar conductas y comportamientos de los padres a sus hijos que pueden catalogarse como más adaptativos o menos adaptativos, en razón de los efectos que tienen sobre sus hijos y en relación con los objetivos que se busquen y la concepción de éxito que se tenga, lo que se sustenta en lo desarrollado en el apartado introductorio. Para efectos de esta reflexión, se pondrá énfasis en la relación padres-hijos y cómo se ha ido trabajando en una escuela de fútbol de una de las sedes de 
un conocido club de Chile. No obstante, también se presentará qué se ha hecho con los niños y el cuerpo técnico de la escuela de fútbol.

El programa cuenta con 60 niños con edades comprendidas entre los 7 y 12 años que fueron seleccionados (grupo de proyección) de las clases de fútbol "abiertas" (cualquier niño puede participar) que hace la escuela de fútbol los días sábado. Estos niños entrenan dos días a la semana en sesiones de 1.5 horas, donde se trabaja de manera más específica las habilidades técnicas y físicas. Este grupo a diferencia del sábado, cuentan con una psicóloga desde Mayo del año 2017, quien se ha incorporado para apoyar la labor de los entrenadores, sin una misión muy específica al momento de su incorporación. Inicialmente, después de una primera reunión con el coordinador general, se detectó necesidades y prioridades, y se propuso un programa psicológico para mejorar la labor de los entrenadores, en aspectos como el manejo de "casos difíciles" y educar en valores a los alumnos (p. ej., respeto y disciplina). Posteriormente, como se comentará más adelante, se amplió el trabajo con los padres y alumnos del grupo de proyección.

\section{Trabajo con los Entrenadores}

Los objetivos básicos que se establecen son los encaminados a apoyar el desarrollo personal y profesional del técnico, ya que es quien pasa mayor tiempo con el deportista y es su principal gestor (García-Naveira y Jerez, 2012). Se apoya también para que su desempeño trascienda lo meramente deportivo y ayuden a fomentar la autonomía y la competencia deportiva del niño.

Se ha abierto un espacio que antes no existía para el área en Psicología del Deporte ni para el rol del psicólogo del deporte (Cantón, 2016), tampoco para poder tratar temas relacionados con aspectos pedagógicos, relacionales, de manejo de situaciones y de gestión de objetivos psicológicos durante el entrenamiento y la competición.

Se organizó una primera reunión con los 4 entrenadores del grupo de proyección de la escuela de fútbol para presentarles los objetivos y acciones del programa. El equipo de trabajo es joven (con una edad entre 23 y 25 años) y hubo bastante apertura, ganas de apoyar y participación. Entre todos, se acordó llamar a este programa: "No todo es balón", ya que el objetivo principal es que los alumnos, además de mejorar a nivel deportivo, pudiesen beneficiarse del deporte como herramienta educacional. Por ello, por una parte, se busca empoderar a los niños en sus propios procesos con orientación a la tarea, es decir, una orientación a la mejoría personal, la autosuperación y la autogestión (estableciendo objetivos a lograr, evaluando cómo pueden mejorar en cada entrenamiento y partido en el que participen), plantear y reflexionar sobre valores importantes como el respeto, esfuerzo, trabajo en equipo, autocuidado y la responsabilidad.

Una vez al mes, de forma grupal, en sesiones de 30-45 minutos cada una, en una sala de las instalaciones deportivas, se organizaba una reunión con los entrenadores para analizar la trayectoria de los alumnos, el rol del entrenador según los objetivos de entrenamiento que ellos establecían y posibles casos individuales a atender. Además, se establecieron ciertas temáticas recogidas como necesidad desde los entrenadores a través de sus experiencias previas y consultadas en la reunión, como el rol educativo, estrategias de apoyo positivo en el deporte y regulación de conductas en entrenamientos y sobre todo en partidos, tanto de los alumnos como del propio entrenador.

Para la educación en valores, se siguió las pautas establecidas por otros autores (Amat y Batalla, 2000; García-Calvo, Sánchez-Oliva, Sánchez-Miguel, Leo, Amado, 2012) en el que destacan: 1) fomentar el autoconocimiento y mejorar el autoconcepto; 2) potenciar el diálogo como mejor forma de resolución de conflictos; 3) la participación de todos; 4) potenciar la autonomía personal; 5) aprovechar el fracaso como elemento educativo; 6) promover el respeto y la aceptación de las diferencias individuales; 7) aprovechar las situaciones de juego, entrenamiento y competición para trabajar las habilidades sociales encaminadas a favorecer la convivencia.

También se plantearon algunos aspectos metodológicos planteados por Escartí, Pascual y Gutiérrez (2005) en su programa de responsabilidad personal y social, como por ejemplo, los formatos diarios de cada sesión de entrenamiento y los niveles de responsabilidad (respeto por los derechos y sentimientos de los demás, participación y esfuerzo y autogestión).

Para realizar un seguimiento, la psicóloga asistía al entrenamiento semanal del grupo de proyección y a un partido al mes por categoría para observar el desarrollo comportamental de los alumnos y de los entrenadores, información que podría ser relevante para próximas reuniones (p. ej., reforzar las conductas deseadas, corregir, dar feedback, etc.).

Otro aspecto que se formalizó con los entrenadores fueron las reuniones técnicas (establecimiento y seguimiento metodológico deportivo), las cuales antes no se realizaban y sólo se iba entregando información de manera informal al término de algunos entrenamientos, del tipo "conversación de pasillo". Se les propuso reunirse mensualmente para evaluar las actividades, ofrecer información de necesidades y plantear objetivos para el mes. Esta era una tarea exclusiva de los entrenadores, en la que se les ayudo a organizar, planificar y desarrollar la actividad. 


\section{Trabajo con los Deportistas}

Antes de comenzar con el trabajo con los alumnos, se hizo una reunión con los padres para explicarles en qué consistiría la incorporación de esta área, de qué trata el programa y se les solicitó una autorización por escrito para realizar actividades formativas y evaluaciones psicológicas con sus hijos. De los sesenta apoderados asistieron 40. De los 20 ausentes se les mandó una comunicación escrita con sus hijos donde tenían que mandar su autorización firmada, la cual era obligatoria para poder participar en la escuela. Todos los padres firmaron la documentación.

Semanalmente, de forma grupal, se realizaban actividades con los alumnos, con una duración entre 20-25 minutos cada una. En un primer encuentro, se reunió a los niños, se hizo la presentación del profesional y éste explicó el rol y función de su presencia, de manera que los niños pudieran incorporarlo y aclarar dudas. En una segunda sesión, para recoger información relevante de ellos, se les aplicó la Escala de Evaluación del Autoconcepto para niños de Piers Harris (1969) adaptado a la población chilena por Gorostegui y Dörr (2004). El objetivo de esta evaluación es poder tener un acercamiento acerca de cómo se ven ellos mismos en distintos aspectos personales (Conducta, Estatus intelectual y Escolar, Apariencia y Atributos Físicos, Ansiedad, Popularidad y Felicidad y Satisfacción).

Una vez que se contó con la aplicación de la escala a todos los niños, se elaboró un informe con los resultados por escala a los padres, el cual fue entregado en segunda reunión con los padres, lo que se explicará en el apartado del trabajo con los padres.

Ya con la presentación y evaluación cumplida, se solicitó a cada niño que trajera un cuaderno con la finalidad de ir escribiendo en él las actividades que se realizarán, tipo bitácora de entrenamiento. Se les entregó un papel impreso al finalizar del entrenamiento para su recordatorio. En este cuaderno, iba pegado en la primera hoja las normas internas del equipo de proyección durante el desarrollo del entrenamiento (cuidar el material y las instalaciones, respetar a compañeros y entrenadores, colaborar en las tareas que se propongan, respetar el horario, justificar inasistencias, obedecer indicaciones del entrenador, etc.) y sugerencias a seguir fuera del horario de entrenamiento y competencia (sacara ropa sucia apenas llegue a casa, cumplir con hábitos de higiene y alimentación, realización de tareas escolares, preparar cosas para el día siguiente, ser responsables de las cosas que llevas y traes). Se solicitó a los niños que estas normas las conversaran en casa con sus padres. El objetivo es que ellos conozcan derechos y deberes y que los padres estén implicados para estar todos en la misma "sintonía".
Estas normas se han ido reforzando al menos 1 vez al mes, haciendo diferentes actividades participativas (p. ej., hacer preguntas a niños elegidos al azar con algún premio).

Si bien aún no ha terminado la temporada deportiva (de marzo a diciembre de 2017), los temas que se tienen planificado y se han ido trabajando en este programa, tanto en las sesiones presenciales y tareas en el cuaderno, son:

- Empoderar a los niños en sus propios procesos con orientación a la tarea. Han ido evaluando sus entrenamientos y partidos con justificación de cómo pueden mejorar en el próximo, se ha elegido el deportista del mes según el esfuerzo, responsabilidad y el trabajo (uno por categoría por edad), explicitando que no el que posee mejor habilidad en el fútbol, sino el que se ha destacado en las características antes mencionadas).

- Reflexionar, incorporar y aplicar valores como el respeto, la valoración del esfuerzo, el trabajo en equipo, el autocuidado, la responsabilidad (a través de role-playing, evaluación de situaciones de partido y entrenamiento, ejercicios de consecución de objetivos en equipo, atención en conductas de burla y fomento de la empatía).

- Trabajar en habilidades como la rapidez en la toma de decisiones, mantención de la atención, control de emociones y la eficacia en la tarea. Se realizaron ejercicios en equipos pequeños de 5-6 alumnos, sobre cómo cumplir con un objetivo que requiere la mantención de la atención, coordinación y delegación de funciones para poder ser los más rápidos y a su vez los más eficientes. El mismo ejercicio se aplicó en 2 oportunidades, con algunas sesiones entremedias donde se explicó la diferencia entre rapidez y eficacia, y hubo cambios de mejora en las respuestas y resultados. También pudieron vivenciar la importancia del trabajo en equipo y el aporte de cada uno, sobre todo aquellos equipos donde algunos participantes se fueron desconcentrando en la tarea.

- Finalmente, dependiendo del tema del mes, se va mandando tarea en el cuaderno con preguntas como: ¿Qué entiendes por trabajo de equipo?, ¿Qué crees que aportas al equipo?, ¿Porqué hacemos el abrazo grupal?, ¿Qué emociones viviste en el partido del fin de semana?, etc.

- Sumado a esto, deben evaluar cada entrenamiento con una nota (de 0, que representa mal, a 10, que significa muy bien) y justificarla, con el objetivo que vayan viendo sus mejoras y lo que pueden mejorar.

Cabe destacar que uno de los primeros hábitos que se enseñó, fue lo que se llamó "el abrazo grupal", con el objetivo de unir, compartir, visualizar objetivos y activarse para la actividad deportiva. Dada la experiencia de la profesional en ejercicios de activación, visualización y respiración, se reúne al equipo (por categoría), se cierran los ojos, se respira 
profundamente, se piensa en el objetivo que se quiere para ese entrenamiento, se lo imaginan, se comprometen con ese objetivo, se respira nuevamente de forma más acelerada y se hace el grito de la escuela. Luego comienza el entrenamiento. Es un ejercicio que por un lado, muestra la finalización del trabajo en el tema específico y da el comienzo al entrenamiento físico-técnico-táctico. Este abrazo grupal con algunas variantes se realiza antes de cada partido. No es necesario que lo coordine el psicólogo, sino que, con supervisión, ya son los propios niños quienes lo van realizando, guiados por algún compañero.

Cómo aún estamos en desarrollo del programa, sólo se han recogido impresiones de los niños de manera informal y a través de las tareas. Sin embargo ya se ve que es un área incorporada, donde, como en todo grupo humano, hay quienes son más responsables o preocupados que otros. Sin embargo, se han recibido felicitaciones por parte de los padres y entrenadores. Al término de la temporada, se aplicará nuevamente la escala de autoconcepto y se pedirá una pequeña evaluación del programa y una autoevaluación.

\section{Trabajo con los Padres}

Se establecieron charlas formales y trimestrales con los padres, en sesiones de 60 minutos aproximadamente cada una. En la primera reunión se tuvo como objetivo presentar el equipo técnico a cargo de los entrenamientos del equipo de proyección, los objetivos a perseguir para este año desde todas las áreas (física, técnico/táctica y psicológica), se presentó también los beneficios que los niños pueden adquirir al participar de una actividad deportiva organizada como este grupo de entrenamiento (habilidades motrices, aprender características técnicas-tácticas del deporte, desarrollo de aspectos psicológicos como autoconfianza, toma de decisiones, aceptar victoria y derrotas, asumir responsabilidades, etc.) y el rol importante que ejercen los padres para el desarrollo no sólo deportivo, si no personal de sus hijos. Por último, se solicitó la autorización de los padres para realizar diferentes actividades y evaluaciones con sus hijos como se ha comentado con anterioridad.

Esta reunión fue citada a través de una comunicación escrita por parte del coordinador de la escuela y enviada la semana anterior, con un recordatorio vía grupo de whatsapp tres días antes del encuentro. La actividad se realizó durante el horario de entrenamiento en un salón ubicado en la recepción del recinto deportivo. Lamentablemente, no asistieron todos los padres (faltaron 20). Esta es un área de mejora para futuras actividades. Algunas justificaciones por ausencia fueron temas de trabajo, viajes y temas domésticos. Sin embargo fue muy productiva, contando con el interés y la participación activa de quienes asistieron.

En la segunda actividad, el procedimiento de convocatoria de los padres fue la misma de la sesión anterior. Contamos con la presencia de la mitad de ellos, con las mismas justificaciones. Lo que ha hecho plantearse la posibilidad de no realizar la reunión en día de semana, sino un sábado en la mañana, aunque sea el día del entrenamiento masivo y no el de proyección.

A los asistentes, se les hizo entrega de los resultados de la evaluación por cada niño de forma personal, a través de un informe, y se explicó de manera general en qué consiste la escala y aspectos generales. La información fue confidencial.

También se le leyó una carta de un hijo a su padre, sacada del libro sobre padres de Peris-Delcampo (2003), donde se explicita ciertas actitudes y estilos educativos de un padre que perjudica o pueden beneficiar la percepción del niño en el deporte. Luego se expuso a través de una presentación lo que pueden hacer los padres para que sus hijos obtengan beneficios de la práctica deportiva, como son (Peris-Delcampo, 2003): asegurarse que la práctica es de calidad a través de la formación de las personas a cargo, la forma de dirigir, objetivos de trabajo, actitud pedagógica; mostrar interés por la práctica deportiva, preguntarle por sus vivencias, sus amigos, si lo pasa bien, centrándose en la experiencia y no en el resultado; colaborar con la escuela en las tareas y todas sus actividades, contestar a tiempo las convocatorias de los niños, asistir a las reuniones; valorar la actividad no sólo por el rendimiento, si no por los beneficios que se pueden conseguir (valores, físicos, disfrute, etc.).

Posterior a esta exposición, se les entregó una hoja con preguntas como: ¿Cuál de las actitudes escuchadas en la carta a veces o muchas veces presento yo? ¿Qué puedo mejorar para que mi hijo obtenga mayores beneficios de la práctica deportiva y valore el esfuerzo y su mejora personal por sobre el resultado? Me comprometo a...

Además se ha ido definiendo comportamientos y zonas para estar de los padres durante la actividad deportiva (antes se colocaban al lado del entrenador o en cualquier lugar de la cancha). El objetivo de esto es que los niños sólo reciban instrucciones de los entrenadores, que los padres disfruten del partido y no se preocupen por los errores. Los padres siguen dando instrucciones, pero ya hay mayor dificultad para ello y los niños van aprendiendo a escuchar indicaciones tácticas del entrenador por sobre los padres, quienes se han ido autorregulando, ya sea por la distancia física o la información entregada sobre ello o ambos.

También se ha enviado físicamente y a través de canales de comunicación como whatsapp algunos informativos y pautas de comportamiento que entregan información so- 
bre la importancia de ser modelos de actuación, de valorar positivamente el error como una forma de aprendizaje, de cómo poder retroalimentar a los niños de manera constructiva, etc. Es decir la entrega de pautas concretas que ayuden al desarrollo de la autoestima y la concepción del éxito en base a la mejoría personal.

También se mejoró el canal de comunicación a través de la creación de un newsletter bimensual con información de partidos y actividades y algunas frases sobre la importancia de la orientación a la tarea y el esfuerzo por sobre el resultado, el fair play, desarrollo de autonomía, ver los errores como aprendizaje, etc.

Fuera de las actividades regladas trimestrales, dentro de las actividades de "hacerse cargo" y responsabilizarse de llevar a cabo sus propias actividades y vivir los beneficios que ello conlleva, se realizaron 2 actividades que solicitamos apoyo de los padres. Una fue una rifa (sorteo) que tuvo como finalidad juntar dinero para cambiar los uniformes de entrenamiento. Anteriormente en situaciones similares, no había una actividad que tuviera un objetivo común (cambiar uniformes o pagar inscripción del campeonato), sólo se solicitaba el dinero a los padres y se realizaba la acción. Sin embargo, dado este programa, quisimos darle más sentido al esfuerzo y compromiso para conseguir objetivos. La mayoría de los padres se sumaron de manera positiva, sin embargo hubo algunos casos que no participaron y simplemente mandaron el dinero.

Otra actividad enmarcada en el desarrollo de la empatía, respeto y valoración de lo que se tiene, fue una campaña solidaria hacia un club de fútbol de la misma comuna, pero de escasos recursos y de niños vulnerables socialmente, denominada: "Ganamos todos". El objetivo era reunir zapatos y zapatillas de fútbol, calcetines, canilleras y guantes y culminar con un partido amistoso donde se entregarían lo recaudado y se haría una convivencia entre los equipos. La campaña tuvo muy bien recibimiento en un principio contando con muchas donaciones y buena disposición, hasta que se puso la fecha del partido amistoso donde hubo muy baja participación por parte de los padres, lo que se reflejó en ausencias de los niños. Finalmente se pudo hacer igual la actividad, pero con menos colaboración de la esperada, con justificaciones como la falta de tiempo y la realización de otras actividades.

Se puede decir en razón a lo observado, que los niños que más participan de las actividades como las mencionadas, los que más asisten a partidos y los que los que en la evaluación de autoconcepto mostraron promedio alto y alta autoestima son los que cuentan con padres que participan de manera activa, sin mostrar mayor presión y que tienen una actitud positiva en entrenamientos y partidos, asumiendo lo que se les solicita. A su vez, son los que participan de las reuniones y de las distintas actividades propuestas.

Por último, aún falta la realización de una última reunión para poder cerrar el año y evaluar el programa desde la mirada de los padres. Sin embargo, se han recibido algunos comentarios positivos por parte de los padres, entrenadores y el coordinador deportivo, así como de la observación de conductas y feedback de los alumnos.

\section{Discusión}

En relación al objetivo de este artículo, plantear antecedentes teóricos y prácticos relacionados a la relación parental con la actividad deportiva de sus hijos, se ha podido evidenciar que los padres y otros significativos cumplen un rol importante en el desarrollo deportivo que hay que educar y apoyar para el proceso deportivo integral y saludable de sus hijos.

De acuerdo a lo expuesto, es importante considerar los contextos sociales, educativos y familiares que van permitiendo el desarrollo de conductas que ayudan a mantenerse y comprometerse en la actividad entre los más jóvenes (Jones et al., 2009). Y si el contexto deportivo es organizado, estructurado y supervisado genera resultados positivos en sus participantes (Whitt-Glover et al., 2009; Zarret et al., 2009; García-Naveira y Jerez, 2012).

Con respecto a los padres, se ha evidenciado que los comportamientos y actitudes de éstos, están asociadas a autopercepciones de habilidad, orientaciones motivacionales, disfrute, satisfacción y comportamientos en el deporte y la educación física, concordando con investigaciones como las de Smith et al. (2009) y Sanchez et al. (2013).

A su vez, investigaciones han mostrado que el apoyo y la comprensión de los padres favorecen el compromiso deportivo y la diversión de los futbolistas con lo que se disminuye la probabilidad del abandono deportivo (Torregrosa et al., 2007). Lo que corrobora con la práctica donde se ha podido evidenciar cómo las actitudes de los padres influyen y se relacionan con la participación deportiva.

Se puede decir, por tanto, en base con la literatura científica, que el contexto influye en un deportista en cuanto a su orientación motivacional y su desarrollo personal. Esa influencia es dada por la forma cómo ese contexto es interpretado subjetivamente en cuanto a criterios presentes de éxito y fracaso y al importancia del esfuerzo (Amblard y Leite, 2015). También influye en las distintas actitudes y desarrollo de autoconcepto y autoestima. Esto va en la línea de Kadushin (2011) y su estudio, donde encontró que los participantes fueron algo más propensos a definir el éxito de una ma- 
nera que fue congruente con el clima motivacional creado por ambos padres y entrenadores. Por otra parte, Sánchez et al. (2013) observaron en su investigación que cuanto mayor fue la disposición de orientación a la tarea de los padres y comportamientos de apoyo, mayor fue esta motivación (a la tarea) en sus hijos. Esto se buscará validar a través de la experiencia con la evaluación final del programa al término de la temporada.

Considerando la etapa formativa, esta área de trabajo debe apoyar y reforzar a los padres para que no olviden la importancia de compaginar estudios y deporte, colaborar con la escuela deportiva o club en sus actividades y decisiones y alabar progresos como una manera de reforzar positivamente el valor del esfuerzo como medida de autosuperación y fuerza interna que llevará a conseguir los objetivos. Concordando con otras experiencias en relación con las reacciones adecuadas ante ante los errores: lo propicio sería animar y valorar el esfuerzo, considerando que sus hijos aún se encuentran en un período de aprendizaje.

Se refuerza, por tanto, la importancia de los adultos implicados en la práctica deportiva en donde un clima enfocado por éstos sólo hacia el resultado, mermaría el disfrute en la actividad y por tanto estaría potenciando el abandono. En este sentido se ha podido ver en la práctica e investigación, que una orientación a la tarea, es decir, valorar el esfuerzo y la mejoría personal por sobre la comparación con otros es positiva, no obstante es importante señalar que la percepción de orientación al ego o el resultado no se encuentra ausente en el deporte, lo que concuerda con algunos autores, acerca de la relación de ego con percepción de competencia, es decir, un grado de foco hacia el resultado proporciona la sensación y creencia que es posible realizar y conseguir el resultado propuesto, siempre y cuando este sea realista.

Una de las actividades definida anteriormente, la implementación del cuaderno por alumno, tuvo en general muy buen recibimiento por parte de los padres, sin embargo contamos con un caso donde la madre se acercó a hablar que no estaba de acuerdo ya que los niños están muy recargados en la escuela y que ella no tiene el tiempo para sentarse ni recordarle a su hijo que se haga responsable del cuaderno. Ante esta situación, simplemente se limitó a decirle que son actividades voluntarias donde tanto su hijo como ellos como padres son los beneficiados, pero que son libres a sumarse o no. Son cosas que pueden pasar, sólo se limitó a seguir reforzando a los niños a través de las sesiones de entrenamiento y mucho apoyo por parte de los entrenadores para que los niños también lo incorporaran como parte del entrenamiento.

Se puede plantear como conclusión que a través del desarrollo de este trabajo, que en lo teórico y en la práctica se puede observar la importancia del rol y de ciertas conductas de los padres por sobre otras para apoyar el desarrollo integral de los hijos, potenciar el disfrute y los beneficios de realizar una práctica deportiva adecuada. Para que los niños puedan contar con un entorno propicio generado por los adultos significativos.

Próximas investigaciones pueden enfocarse precisamente en indagar en las presiones que se ejercen y que contribuyen o afectan a las conductas de los padres y éstas a la percepción de disfrute de los practicantes. Analizando los porcentajes de participación a principio de la temporada $y$ al final.

Finalmente como proyecciones a futuro, se podrían realizar trabajos de corte intervencionista en los que se consideren a estos adultos significativos en un trabajo a largo plazo y con seguimiento, pudiendo examinar la interacción entre la madre, padre y entrenador, en la creación de clima motivacionales y colectivamente pre y post intervención. También examinar cómo estas características socio-ambientales ayudarían a predecir las intenciones futuras de participación deportiva.

\section{Referencias}

Amat, M. y Batalla, A. (2000). Deporte y Educación en valores. Aula de Innovación Educativa, 91, 10-13.

Amblard, I. y Leite, F. (2015). Sentidos de Vitória/Derrota para os Pais Segundo Atletas do Alto Rendimiento. Psicologia: Ciência É Profissao, 35, 643-658.

Ames, C. (1992). Achievement goals, motivational climate, and motivational processes. In G.C. Roberts (Ed.), Motivation in Sport and Exercise (pp. 161-176). Champaign, IL, Estados Unidos: Human Kinetics.

Baumrind, D. (1971). Current pattern of parental authority. Developmental Psychology, 4(1), 2-4.

Cantón, E. (2016). La especialidad profesional en Psicología del Deporte. Revista de Psicología aplicada al Deporte y al Ejercicio Físico, 1, e2. https://doi.org/10.5093/rpadef2016a2

Castillo, I., Duda, J. L., Álvarez, M. S., Mercé, J., y Balaguer, I. (2011). Clima motivacional, metas de logro de aproximación y evitación y bienestar en futbolistas cadetes. Revista de Psicología del Deporte, 20(1), 149-164.

Cerezo, M.T., Casanova, P.F., De la Torre, M.J. y De la Villa, M. (2011). Estilos educativos paternos y estrategias de aprendizaje en alumnos de Educación Secundaria. European Journal of Education and Psychology, 4(1), 51-61.

Cervelló, E., Escartí, A., y Balagué, G. (1999). Relaciones entre la orientación de meta disposicional y la satisfacción con los resultados deportivos, las creencias sobre las causas de éxito en deporte y la diversión con la práctica deportiva. Revista de Psicología del Deporte, 8(1), 7-19.

Duda, J. L. y Balaguer, I. (2007). The coach-created motivational climate. En S. Jowett y D. Lavalee (Eds.), Social Psychology of Sport (pp. 117-130). Champaign, IL, Estados Unidos: Human Kinetics 
Escartí, A., Pascual, C. y Gutiérrez, M. (2005). Responsabilidad personal y social a través de la educación física y el deporte. Barcelona, España: Biblioteca de Tándem.

Fredericks, J. y Eccles, J. (2006). Extracurricular involvement and adolescent adjustment: Impact of duration, number of activities, and breadth of participation. Applied Developmental Science, 10, 132-146.

García-Calvo, T., Sánchez-Oliva, D., Sánchez-Miguel, P. A., Leo, F. M., Amado, D. (2012). Escuela del deporte: valoración de una campaña para la promoción de valores. European Journal of Human Movement, 28, 67-81.

García-Ferrando, M. (2006). Veinticinco años de análisis del comportamiento deportivo de la población española (1980-2005). Revista Internacional de Sociología, 44, 15-38.

García-Mas, A., Palou, O., Smith, R. E., Ponseti, X., Almeida, P., Lameiras, J. y Leiva, A. (2011). Ansiedad competitiva y clima motivacional en jóvenes futbolistas de competición, en relación con las habilidades y el rendimiento percibido por sus entrenadores. Revista de Psicología del Deporte, 20(1), 197-207.

García- Naveira, A. y Jerez, P. (2012). Departamento de Psicología del club Atlético de Madrid: filosofía, Programación y desempeño profesional en el fútbol base. Cuadernos de Psicología del Deporte, 12(1), 111-120

Gómez-López, M., Granero-Gallegos, A., Baena-Extremera, A., y Abraldes, J. A. (2014). Goal orientation effects on elite handball players motivation and motivational climate. Procedia - Social and Behavioral Sciences, 132, 434-440.

Gorostegui, M. E, Dörr, A. (2004). La Escala de Evaluación del Autoconcepto para Niños de Piers Harris: Actualización de Normas. Castalia Revista de Piscología de la Academia, 7, 81-97.

Jones, M., Dunn, J., Holt, N., Sullivan, P y Bloom, G. (2009). Exploring the " $5 \mathrm{Cs}$ " of positive youth development in sport. Journal of Sport Behavior, 34, 250-267.

Kadushin, P. (2011). Perceptions of Parent-Initiated and Coach-Created Motivational Climate and their influence on youth athlete achievement goal orientation. (Tesis de Máster) ProQuest Dissertations and Theses Data Base (UMI Number: 1504903)

Määttä,S., Ray, C. y Roos, E. (2014). Associations of parental influence and 10-11-year-old children's physical activity: Are they mediated by children's perceived competence and attraction to physical activity? Scandinavian Journal of Public Health, 42, 45-51.

Merino, A., Arraiz, A. y Sabirón, F. (2017). La Adherencia del entorno familiar en el fútbol prebenjamín: un estudio de caso. Revista Iberoamericana de Psicología del Ejercicio y del Deporte, 12(1), 139-148

Newton, M., Duda, J. L. y Yin, Z. (2000). Examination of the psychometric properties of the Perceived Motivational Climate in Sport Questionnaire-2 in a simple of female athletes. Journal of Sports Sciences, 18, 275-290.

Nicholls, J. G. (1989). The competitive ethos and democratic education. Cambridge, MASS, Reino Unido: Harvard University Press.

O’Rourke, D., Smith, R., y Smoll, F. (2014). Relations of parent-and coach initiated motivational climates to young athletes'self-esteem, performance anxiety and autonomous motivation: who is more influential? Journal of Applied Sport Psychology, 26, 395408.

Ortiz, P. (2016) Compromiso deportivo y clima motivacional en jóvenes futbolistas. Granada, España: Editorial Publicia.
Ortiz, P., Chirosa, L., Martín, I., Reigal, R. E. y García- Mas, A. (2016). Predicción del Compromiso Deportivo a través de la percepción del Clima Motivacional de madre, padre y entrenador, en futbolistas jóvenes. Revista de Psicología del Deporte, 25, 245-252.

Peris-Delcampo, D. (2003). Los Padres son importantes... Para que sus hijos hagan deporte. Catellón, España: Editorial. Fundación C.D. Castellón.

Quested, E., Ntoumanis, N., Viladrich, C., Haug, E., Ommundsen, Y., Van Hoye, A.,... Duda, J. (2014). Intentions to drop-out of youth soccer: A test of the basic needs theory among European youth from five countries. International Journal of Sport and Exercise Psychology 11, 395-407.

Quested, E. y Duda, J. L. (2011). Enhancing children's sport experiences and personal development: A motivational perspective. En R. Bailey y I. Stafford (Eds.), Coaching children in sport (pp. 123-138). London, Reino Unido: Routledge.

Roberts, G. C. (2001). Advances in Motivation in sport and exercise. Champaign, IL, Estados Unidos: Human Kinetics.

Roberts, G. C. y Treasure, D. (2012). Advances in motivation in sport and exercise (3rd ed.). Leeds: Human Kinetics.

Sánchez, P., Leo, F., Sánchez-Oliva, D., Amado, D. y Gracía-Calvo, T. (2013). The importance of parents'behavior in their children's enjoyment and amotivation in sports. Journal of Human Kinetics, 36, 171-179.

Santi, G., Bruton, A., Pietrantoni, L. y Mellalieu, S. (2014). Sport commitment and participation in master swimmers: The influence of coach and teammantes. European Journal of Sport Science, 14, 852-860.

Saybani, H., Yusof, A., Hassan, A. y Zardoshtian, S. (2013). Athlete's Satisfaction as Mediator of Transformational Leadership Behaviors of Coaches and Football Players'Sport Commitment Relationship. World Applied Sciences Journal, 21, 1475-1483.

Smith, R. E., Cumming, S. P. y Smoll, F. L. (2008). Development and validation of The Motivational Climate Scale for Youth Sports. Journal of Applied Psychology 20(1), 116 - 136.

Sousa, C., Torregrosa, M., Viladrich, C., Villamarín F. y Cruz, J. (2007). The commitment of young soccer players. Psicothema 19, 256-262.

Taliaferro L., Rienzo B. y Donovan K. (2010). Relationships between youth sport participation and selected health risk behaviors from 1999 to 2007. Journal of School Health, 80, 399-410.

Torregrosa, M., Chamorro, J. y Ramis, Y. (2016). Transición de junior a senior y promoción de carreras duales en el deporte: una revisión interpretativa. Revista de Psicología Aplicada al Deporte y al Ejercicio Físico, 1, e6. https:// doi.org/10.5093/rpadef2016a6

Torregrosa, M., Cruz, J., Sousa, C., Vildarich, C., Villamarín, F., Garcia- Mas, A. y Palou, P. (2007). La influencia de padres y madres en el compromiso deportivo de futbolistas jóvenes. Revista Latinoamericana de Psicología, 39, 227-237.

Veskovic, A., Valdevit, Z. y Dordevic-Nikic, M. (2013). Goal orientation and perception of motivational climate initiated by parents of female handball players of different competition levels. Facta Universitatis, Serie: Physical Education and Sport, 17, 337-345.

Wentzel, K. (1998). Social relationships and motivation in middle school: The role of parents, teachers, and peers. Journal of Educational Psychology 90, 202-209.

Whitt-Glover, M., Taylor, W., Floyd, M., Yore, M., Yancy, A. y Matthews, C. (2009). Disparities in psysical activity and sedentary 
behavooir among US children and adolscents: prevalence, correlates and intervention implications. Journal of Public Health Policy, 30, 309-333.

Wuerth, S., Lee, M. y Alfermann, D. (2004). Parental involvemen and athletes' career in youth sport. Psychology of Sport and Exercise, 5, 21-33.
Zarret, N., Fay, K., Li, Y., Carrano, J., Phelps, E. y Lerner, R. (2009). More tan child's play: Variable-and pattern-centered approaches for examining effects of sports participation on youth development. Developmental Psychology, 45, 368-382. 